\title{
El Seminario Internivelar de Evaluación Formativa y Compartida en Educación.
}

\section{The inter-level seminar of Formative and Shared Assessment in Education}

\author{
Cristina Pascual Arias ${ }^{1}$, Víctor M. Lopez-Pastor ${ }^{2}$ \& Carolina Hamodi Galán ${ }^{3}$
}

\begin{abstract}
Fecha de recepción: 24/01/2019; Fecha de revisión: 23/02/2019; Fecha de aceptación: 25/03/2019 Cómo citar este artículo:

Pascual-Arias, C., López-Pastor, V. M., \& Hamodi, C. (2019). Proyecto de Innovación Docente (PID) y Seminario Permanente internivelar sobre Evaluación Formativa y Compartida. Transferencia de conocimiento entre universidad y escuela. Revista de Innovación y Buenas Prácticas Docentes, 8, 27-34. Autor de Correspondencia: cristina.pascual@uva.es
\end{abstract}

\section{Resumen:}

En este artículo presentamos el desarrollo de un seminario de Formación Permanente del Profesorado en Evaluación Formativa y Compartida, con profesorado de todas las etapas educativas (desde Educación Infantil hasta Educación Universitaria). Es una de las actividades principales del Proyecto de Innovación Docente "la evaluación formativa y compartida en Educación. Transferencia de conocimiento entre Universidad y Escuela", que se desarrolla actualmente en la Facultad de Educación de Segovia (Universidad de Valladolid). Mostramos el desarrollo de esta experiencia durante el curso 2017/2018, en la que los maestros desarrollan prácticas de Evaluación Formativa y Compartida en sus aulas que van reconduciendo a través de ciclos de Investigación-Acción. En este artículo mostramos las etapas por las que fue pasando el seminario durante el curso 2017/2018. Los primeros resultados obtenidos señalan que todas las experiencias desarrolladas hacen un análisis de ventajas, inconvenientes y propuestas de mejora que se están llevando a cabo durante el presente curso 2018/2019.

Palabras clave: Evaluación Formativa, Evaluación Compartida, Transferencia de Conocimiento, Formación Permanente del profesorado.

\begin{abstract}
:
In this paper we present the development of a inter-classroom seminar of In-Service Teacher Education in Formative and Shared Assessment in all stages of education (from early childhood Education to University). It is one of the main activities of the Teaching Innovation Project "the Formative and Shared Assessment in Education. Transfer of knowledge between University and School", which is currently carried out in the Faculty of Education of Segovia (University of Valladolid). We show the development of this experience during the 2017/2018 academic year, in which the teachers develop practices of Formative and Shared Assessment in their classrooms that they are redirecting through Action-Research cycles. In this article we show the stages that the seminar was going through during the 2017/2018 academic year. We show the first result obtained, noting that experiences have been developed in all educational stages with advantages, disadvantages and improvement proposal that are taking during the course of the current academic year 2018/2019.

Key Words: Formative Assessment, Shared Assessment, Knowledge Transfer, In-Service Teacher Education
\end{abstract}

\footnotetext{
1 Universidad de Valladolid, Facultad de Educación de Segovia (España), cristina.pascual@uva.es; CÓDIGO ORCID: 0000-0002-2781-5600

2 Universidad de Valladolid, Facultad de Educación de Segovia (España), vlopez@mpc.uva.es; CÓDIGO ORCID. 0000-0003-2681-9543

3 Universidad de Valladolid, Facultad de Educación de Soria (España), carolina.hamodi@uva.es; CÓDIGO ORCID. 0000-0002-0965-8410
} 


\section{INTRODUCCIÓN}

La experiencia de innovación educativa que presentamos se centra en la creación y funcionamiento de un Seminario Internivelar que centra su trabajo en la temática de la Evaluación Formativa y Compartida (EFyC) en educación. Lleva funcionando desde septiembre de 2017, ligado a la Facultad de Educación de Segovia. Se trata de un Seminario de Formación Permanente del Profesorado, abierto a la participación del profesorado de todas las etapas educativas (Educación Infantil, Primaria, Secundaria y Universidad) interesado que trabaja en esta temática. El seminario forma parte de un proyecto de innovación docente (PID) más complejo, denominado "La evaluación formativa y compartida en educación. Transferencia de conocimiento entre Universidad y Escuela", que se está desarrollando en tres campus de la Universidad de Valladolid (Campus de Segovia, Soria y Valladolid), durante los cursos 2017-2018 y 2018-2019.

El funcionamiento del PID supone una innovación docente al desarrollar la transferencia de conocimiento en dos líneas de intervención: (a) desde la universidad a los centros educativos; (b) desde los centros educativos a la universidad. EI PID ayuda a acercar al alumnado de Formación Inicial del Profesorado (FIP), los futuros maestros y maestras, a la realidad de los colegios, conociendo en profundidad que prácticas son consideradas de excelencia y realizando diferentes actividades de aprendizaje sobre Evaluación Formativa y Compartida (EFyC): Proyectos de Aprendizaje Tutorado (PATs), periodos de Prácticum, Trabajos Fin de Grado (TFG), Trabajos Fin de Máster (TFM) y Tesis Doctorales.

El seminario de Formación Permanente del Profesorado, está basado en el desarrollo de aprendizajes de EFyC que los docentes que participan en él llevan a cabo a través de ciclos de Investigación-Acción (I-A). Esta modalidad de formación permanente tiene una larga trayectoria en educación en los últimos años, consiguiendo muy buenos resultados educativos. Muestra de ello es el trabajo que se expone en Hamodi, López \& López (2014), donde destacan entre las actividades voluntarias que lleva a cabo la Red de Evaluación, la implantación en seminarios de formación permanente en educación superior realizados a nivel local, para compartir experiencias y reflexiones sobre la práctica docente en la etapa Universitaria. Este seminario desarrollado años atrás sobre la práctica evaluativa en Educación Superior, les ayudó a resolver problemáticas, recibir información y recursos, así como compartir experiencias y conocimiento profesional. La trayectoria de la modalidad de formación permanente la avalan autores como Hamodi, López \& López (2014), Barba-Martín, Barba \& Martínez (2016), destacando el aprendizaje de los participantes a colaborar en grupo, a mejorar la autonomía y la predisposición positiva hacia la formación permanente.

La metodología que tienen en común todas estas experiencias de formación permanente del profesorado son los ciclos de I-A que se llevan a cabo. Podemos encontrar trabajos (López et al., 2016; López, Monjas \& Manrique, 2011), que recogen el trabajo del Seminario Permanente de Educación Física de Segovia, que lleva funcionando desde hace 25 años. Esta metodología genera procesos de reflexión que ayudan a mejorar la práctica docente e influyen positivamente en su desarrollo profesional. En López et al. (2016) y Córdoba et al. (2016) nos muestran la importancia del aprendizaje cooperativo en la Formación Permanente del Profesorado; el apoyo mutuo entre docentes les permite poner en común experiencias y reflexiones de cada miembro o grupo de trabajo, llegando así a una reflexión conjunta y creación de conocimientos compartidos.

\subsection{La Evaluación Formativa y Compartida y como práctica de cambio educativo}

El hilo conductor del seminario es la EFyC como práctica de cambio educativo. Según López-Pastor \& Pérez-Pueyo, (2017, p.36) la "Evaluación Formativa" es: "todo proceso de evaluación cuya finalidad principal es mejorar los procesos de enseñanzaaprendizaje que tienen lugar. Sirve para que el alumnado aprenda más (y/o corrija sus 
errores) y para que el profesorado aprenda a trabajar mejor (a perfeccionar su práctica docente). De esta manera la evaluación formativa debe servir para mejorar en tres sentidos: (1) el proceso de aprendizaje del alumnado; (2) la competencia docente; (3) el proceso de enseñanza aprendizaje en sí mismo". López-Pastor, \& Pérez-Pueyo (2017, p.45) definen la "Evaluación Compartida" como "procesos de diálogo que mantiene el profesor con su alumnado sobre la evaluación de los aprendizajes y los procesos de enseñanza-aprendizaje que tienen lugar, pueden ser individuales o grupales y suelen estar basados o relacionados con procesos previos de autoevaluación o evaluación entre iguales".

\subsection{Formación permanente del profesorado e investigación-acción}

El aprendizaje de los docentes del seminario lo realizan a través de ciclos de I-A que llevan a cabo en sus aulas. El proceso de desarrollo de nuestro PID tiene un carácter cíclico, condicionado por la I-A, pues como señala Latorre (2003) el proceso de I-A está compuesto por ciclos y espirales de investigación y acción, constituidos por una serie de fases: (1) planificar, (2) actuar, (3) observar y (4) analizar.

Los ciclos de I-A facilitan mejorar la práctica y aprender de ella. Es decir, reflexionar sobre la práctica llevada a cabo para volver a planificar y mejorar de manera permanente el proceso de enseñanza-aprendizaje. En este proceso toma también especial relevancia el aprendizaje colaborativo, entendido como el trabajo conjunto del grupo de maestros para conseguir un fin común, en este caso: desarrollar su formación permanente en las competencias profesionales ligadas a la temática de la evaluación formativa y compartida en educación. Como podemos ver en Fraile (2004) y López et al (2016), el interés de esta práctica sobre EFyC reside en la realización de procesos de formación permanente colaborativa con otros compañeros para conseguir una mayor autonomía y desarrollo profesional. A través de dicho aprendizaje colaborativo se ha aportado al profesorado conocimientos para realizar el cambio hacia una EFyC. En consecuencia, enfocamos este aprendizaje cooperativo en la formación permanente del profesorado desde dos perspectivas: (1) el trabajo grupal, que han realizado los docentes durante los seminarios de formación; (2) la tutoría entre iguales, ya que el desarrollo de sus prácticas y sus resultados se han mostrado y analizado en grupo mediante aprendizaje colaborativo.

Hay muchos autores, estudios y experiencias que avalan las ventajas de realizar procesos de Formación Permanente del Profesorado utilizando la I-A como metodología básica de trabajo. Por ejemplo, López et al. (2016) defienden que: (1) la participación en grupos de trabajo de I-A supone un fuerte estímulo y ayuda en el desarrollo profesional docente; (2) parece ser un elemento importante en la mejora de la calidad docente del profesorado implicado en el mismo, así como un fuerte apoyo para ir solucionando problemas que surgen en la práctica educativa; (3) favorece el desarrollo profesional docente y la mejora de la calidad educativa en el área de EF.

Por su parte, Córdoba et al (2016) expone que para generar aprendizajes docentes en los demás compañeros y en uno mismo es necesario difundir y compartir los resultados entre maestros y profesores de diferentes contextos educativos. También pueden encontrarse trabajos en contextos similares que utilizan la I-A en la formación permanente del profesorado, como el proyecto formación permanente de educación inclusiva a través del aprendizaje colaborativo (Barba-Martín, Barba, \& Martínez, 2016), que señalan que a través del trabajo cooperativo entre docentes de diferentes centros se desarrollan procesos de aprendizaje compartido.

Como conclusión, se pueden encontrar experiencias y planteamientos basados en la colaboración y la cooperación entre docentes con una larga trayectoria, que avalan estas ventajas (Barba-Martín, Barba, \& Martínez (2016); Córdoba et al. (2016); 
Fernández-Río, \& Méndez-Giménez (2016); Fraile (2004; Hamodi, López \& López, (2014); López, Monjas, \& Manrique, 2011; López et al., 2016).

\section{DESARROLLO DE LA EXPERIENCIA DE INNOVACIÓN}

La experiencia que presentamos se centra en el denominado "Seminario Internivelar de Segovia sobre Evaluación Formativa y Compartida en Educación". Este seminario se inició el septiembre de 2017 y ha estado funcionando durante todo el curso académico 2017/2018, retomando de nuevo el trabajo a partir de septiembre de 2018, con la intención de volver a funcionar a lo largo de todo el curso 2018-19. Durante el año 2017/2018, se han llevado a cabo 12 reuniones a lo largo de todo el curso, con un desarrollo progresivo tanto de contenidos como de debate. El contenido de dichos seminarios ha quedado reflejado en 12 actas que detallan tanto los participantes asistentes en cada sesión como los temas de debate y acuerdo. A lo largo de ambos cursos el seminario ha pasado y va pasando por las siguientes etapas:

$1^{\circ}$. Formación inicial para los participantes en el seminario permanente (profesorado de colegios, de universidad y estudiantes): se llevó a cabo por especialistas en EFyC mediante un curso inicial de formación y puesta en común de los conocimientos previos. La formación consistió en presentar ideas básicas sobre la EFyC, un análisis colectivo de un dossier de instrumentos de evaluación en casos prácticos concretos y aclaración de dudas.

$2^{\circ}$. Sesiones de trabajo: se llevaron a cabo cada 2-3 semanas con una duración de 60-70 minutos. La estructura de los mismos ha sido la siguiente (con cada componente):

(a) exposición de experiencias y problemas de su sistema de evaluación en el aula, la mayor parte del tiempo los docentes exponen por turnos sus vivencias, lo que ocurre en su sistema de evaluación planificado, los problemas, etc.;

(b) reflexión grupal sobre las experiencias del resto de compañeros o los problemas que surjan en los debates, realizando indicaciones o propuestas para mejorar la práctica docente;

(c) concreción del plan de acción y fecha de la siguiente reunión. Todos los debates, exposiciones, aclaraciones, consensos, etc., de cada reunión quedan reflejados en el acta de cada sesión correspondiente. De esta manera, desarrollamos tres procesos cognitivos que dan paso al desarrollo de conocimientos compartidos (Echeita, 1995, p.180): (1) la colaboración, a través de la cual los docentes construyen un boque común de conocimientos y procedimientos; (2) la regulación, a través del lenguaje; en las reflexiones grupales cada maestro expresa sus experiencias y regula sus acciones, a la vez que es guiado por el lenguaje del compañero; (3) se producen las controversias, se analizan las diferencias, los debates entre diferentes perspectivas pasando por procesos de colaboración entre los participantes para desarrollar procesos de reflexión grupal y apoyo formativo. Por tanto, se han desarrollado ciclos encadenados de investigación-acción, que consta de las siguientes fases: (a) planificación del sistema de EFyC a utilizar en sus aulas; (b) puesta en práctica de lo planificado; (c) observar y recoger datos sobre los resultados obtenidos; (d) analizar y reflexionan sobre lo acontecido para comenzar con la planificación y un nuevo ciclo de I-A, de forma que permita una mejora progresiva de los sistemas de evaluación puestos en práctica.

$3^{\circ}$. Vinculación de estudiantes universitarios al proyecto: durante el año 2017/2018 se realizaron dos tesis doctorales asociadas al seminario, vinculadas con los procesos de aprendizaje colaborativo en el seminario de EFyC. También se desarrollaron 1 TFG y 1 TFM sobre la EFyC y su puesta en práctica en contextos escolares reales. En el curso actual, las dos tesis siguen vinculadas al proyecto, y se 
realizarán 2 TFG más sobre aplicaciones concretas de sistemas de EFyC durante las prácticas en educación infantil y educación primaria.

$4^{\circ}$. Jornada final de intercambio de experiencias. Tuvo lugar en el campus María Zambrano (Segovia), en el mes de junio de 2018. Se organizó conjuntamente con el PID sobre Educación Inclusiva que se había desarrollado de manera paralela en el mismo campus durante todo el curso 2017-18. Tuvo como objetivo explorar las posibilidades de una evaluación formativa inclusiva y el intercambio de experiencias desarrolladas sobre EFyC. Se compuso de: conferencias a cargo de expertos externos al grupo; exposición de actividades llevadas a cabo; talleres de formación y mesas redondas sobre experiencias acumuladas. En el presente curso, 2018/2019, se incluirá dentro del XII Congreso Internacional de Evaluación Formativa y Compartida en Educación, que se llevará a cabo en Segovia los días 1, 2 y 3 de julio de 2019. Dentro de este congreso se realizarán dos ponencias magistrales, mesas redondas, mesas de debate, exposición de comunicaciones sobre diferentes temáticas de EFyC y talleres prácticos sobre EFyC en diferentes etapas.

$5^{\circ}$. Difusión de resultados: los participantes en el seminario y el alumnado que está realizando sus TFG, TFM y tesis doctorales sobre el tema han presentado diferentes trabajos y comunicaciones en diversos congresos especializados sobre la temática con los resultados encontrados. Al finalizar el curso 2017/2018 expusimos los resultados del seminario en el "XI Congreso Nacional de Evaluación Formativa y Compartida. La evaluación educativa: entre la emoción y la razón" (Pascual-Arias, López-Pastor, \& Hamodi, en prensa); así como en el "XI Congreso Internacional de Actividades Físicas Cooperativas" (Pascual, Molina, Fernández, \& Fuentes, 2018). Durante este curso, 2017/2018 difundiremos los resultados en el capítulo de un libro centrado en recopilar prácticas de éxito en evaluación en docencia universitaria que será publicado próximamente.

\section{RESULTADOS}

En este apartado vamos a presentar los principales resultados alcanzados por el Proyecto de Innovación Docente durante el curso 2017-18, dado que es el único que se ha completado hasta el momento. Durante el actual curso 2018-19 el proyecto sigue funcionando básicamente igual, con la incorporación de nuevos maestros al seminario.

Los resultados que destacamos del desarrollo del proyecto durante el curso 2017/2018 son muy positivos. Los detallamos a continuación:

$1^{\circ}$. El seminario de formación permanente ha supuesto una innovación, ya que se han desarrollado experiencias de EFyC en todas las etapas educativas. Desde Educación Infantil hasta Educación Universitaria pasando por Educación Primaria y Secundaria. Habitualmente el profesorado de los colegios pierde el hábito de compartir sus experiencias docentes y reflexionar de manera colectiva y cooperativa sobre las mismas. Así mismo, tampoco suele contar con espacios donde encuentren expertos en temáticas (como la EFyC) y pueda ser asesorado sobre su quehacer docente. Gracias a la participación de 21 docentes de todas las etapas educativas hemos visto como el desarrollo de EFyC es posible adaptado a cada contexto, cada nivel y cada grupo/clase durante el curso 2017/2018 (ver tabla 1):

Tabla 1.

Número de participantes y etapa educativa durante el seminario en el curso 2017/2018.

\begin{tabular}{lc}
\hline \multicolumn{1}{c}{ Etapa educativa } & Número de participantes \\
\hline Educación Infantil & 1 \\
Educación Primaria & 9 \\
Educación Secundaria & 1 \\
Educación Universitaria & 10 \\
\hline
\end{tabular}


Todos los participantes del seminario desarrollaron el informe de evaluación de la Red de Evaluación Formativa y Compartida. Existen tres tipos de informe que cada docente escoge adecuándose a su etapa educativa: (a) etapa no universitaria; (b) etapa universitaria sencillo, sin cuestionarios; $y$, (c) etapa universitaria complejo con resultados de cuestionarios anónimos de los alumnos).

Los informes tienen unas partes similares que han ido realizando en el trascurso del seminario, quedando la elaboración del informe dividido en dos partes:

1. En la primera parte del curso se realizó la parte de planificación sobre su proceso de EFyC (definición del contexto, explicación del sistema de evaluación y calificación, actividades de aprendizaje con su correspondiente evaluación formativa y salto a la calificación).

2. En la segunda se terminó de realizar el informe plasmando los resultados a los que se ha llegado en cada caso (resultados-efecto del rendimiento académico, interpretación de los resultados, ventajas encontradas, inconvenientes, propuestas de mejora y conclusiones).

Los informes se corrigieron de manera progresiva con el coordinador de dicho seminario a través de feedback mantenido mediante correo electrónico y las reuniones presenciales de seminario.

Todos los miembros del seminario reflejan que gracias al sistema de EFyC que han desarrollado en sus clases el proceso de enseñanza-aprendizaje ha mejorado. Los alumnos ahora son conscientes de su propio aprendizaje, como docentes han podido mejorar su práctica y el proceso en si mismo ha sido enriquecido a partir de la propia EFyC. También reflejan ventajas e inconvenientes sobre el proceso de EFyC que han desarrollado. Todos los inconvenientes que se han reflejado llevan adjunta su propuesta de mejora para años sucesivos. Por lo tanto, estamos ante un proceso en el que los propios docentes ven los puntos débiles de su puesta en práctica y proponen mejoras para solventarlos. Como último resultado a destacar, señalamos la continuidad del seminario durante el presente curso 2018/2019 por parte de la mayoría de los docentes que lo formaron el curso anterior, así como la ampliación de este grupo con más profesionales de todas las etapas educativas.

$2^{\circ}$. La doble transferencia de conocimiento: por un lado, el profesorado del colegio mejora su práctica docente a partir de su participación en actividades formativas realizadas en la universidad y a través de su colaboración en el periodo de prácticas y en desarrollo de trabajos académicos, y el profesorado y alumnado de la facultad de educación mejora su formación gracias al contacto con la realidad de los centros educativos.

$3^{\circ}$ - Otro resultado a considerar es, la vinculación del seminario con un TFG y un TFM durante el curso 2017/2018, así como con una Tesis Doctoral. El desarrollo del TFG obtuvo unos resultados óptimos por parte de la maestra en prácticas, tanto para su formación docente como para el desarrollo de su TFG. También en el caso del TFM, la proyección y desarrollo del trabajo fue óptimo, obteniendo unos resultados muy satisfactorios. Tanto en el TFG como en el TFM y en la Tesis Doctoral, la vinculación con el seminario ha servido de fuente de datos y aprendizajes para avanzar y enriquecer dichos trabajos.

\section{CONCLUSIONES}

El seminario de formación permanente sobre EFyC ha funcionado adecuadamente a lo largo del curso 2017-2018 y sigue funcionando durante el actual curso 2018/2019. En las experiencias de EFyC desarrolladas los docentes han adquirido conocimientos nuevos sobre procesos de EFyC y están mejorando sus técnicas de una manera y progresiva.

El desarrollo del seminario ha sido muy satisfactorio desde todos los puntos de vista (aprendizaje, contenido, implicación, formación...), lo que está permitiendo seguir mejorando a partir del camino andado durante el presente curso. El aprendizaje entre 
los docentes es muy satisfactorio, ya que las reuniones de seminario nos permiten compartir dudas y problemas y proponer soluciones entre los propios profesionales docentes. Gracias a la EFyC han mejorado su práctica docente individual y se ha creado un espacio de aprendizaje colaborativo que les ha dado mucho respaldo profesional. De todas las experiencias que se han llevado a cabo en el seminario, 9 de ellas se presentaron como comunicaciones XI Congreso de la Red De Evaluación Formativa y Compartida, que se celebró en Córdoba en septiembre del pasado 2018, además de dos comunicaciones sobre el proceso de investigación desarrollado.

En cuanto al desarrollo de trabajos académicos y de investigación, ligados a la realización de TFG, TFM y Tesis Doctorales, podemos concluir diciendo que los resultados han sido muy satisfactorios, porque han vivenciado como alumnos procesos de EFyC en su proceso de Formación Inicial de Profesorado (desarrollo de PAT y procesos de Prácticum) además de adquirir competencias como profesionales que llevan a cabo dichos procesos en contextos reales. Por tanto, han aprendido desde el punto de vista de maestros y alumnos.

Este trabajo puede resultar interesante para docentes de todas las etapas educativas que estén desarrollando o quieran desarrollar prácticas de EFyC. Del mismo modo puede resultar de interés para docentes (universitarios o no) que quieran desarrollar seminarios de formación permanente del profesorado.

Como prospectiva de futuro, intentaremos ampliar las líneas de trabajo de seminario de formación permanente en el resto de provincias que abarca la Universidad de Valladolid, para trabajar de manera paralela a la que se ha desarrollado en el Campus de Segovia.

Sin duda podemos concluir diciendo que gracias a la inserción de prácticas de EFyC ha mejorado el aprendizaje de los alumnos, la capacidad docente de los profesionales y los procesos de enseñanza y aprendizaje, adaptándose a lo que el docente observaba y los propios alumnos demandaban y evaluaban.

\section{REFERENCIAS}

Barba-Martín, R. A., Barba, J. J., \& Martínez Scott, S. (2016). La formación continua colaborativa a través de la investigación-acción. Una forma de cambiar las prácticas de aula. Contextos educativos, 19, 161-175.

Córdoba, J. M. et al. (2016). Educación Física Cooperativa, formación permanente y desarrollo profesional. De la escritura colectiva a un relato de vida compartido. Retos, 29, 264-269. Recuperado de https://recyt.fecyt.es/index.php/retos/article/download/40965/25492

Echeita, G. (1995). El aprendizaje cooperativo. Un análisis psicosocial de sus ventajas respecto a otras estructuras de aprendizaje. En Fernández Berrocal, P. y M. A. Melero (Comps). La interacción social en contextos educativos, (pp. 167-189). Madrid: Siglo XXI.

Fernández-Rio, J., \& Méndez-Giménez, A. (2016). El aprendizaje cooperativo: modelo pedagógico para Educación Física. Retos, 29, 201-206.

Fraile, A. (2004). Didáctica de la Educación Física: una perspectiva crítica y transversal. Madrid: Biblioteca Nueva

Latorre, A. (2003). La investigación acción. Conocer y cambiar la práctica educativa. Barcelona: Graó.

Hamodi, C., López, A. T., \& López-Pastor, V. M. (2014). La Red de evaluación formativa y compartida en docencia universitaria: Creación, consolidación y líneas de trabajo. Revista de Evaluación Educativa, 3(1). Recuperado de http://revalue.mx/revista/index.php/revalue/issue/current

Kemmis, S., \& McTaggart, R. (1988). Cómo planificar la investigación-acción. Barcelona: Laertes 
López-Pastor, V. M. et al., (2016). Veinte años de formación permanente del profesorado, investigación-acción y programación por dominios de acción motriz. Retos, 29, 270-279.

López-Pastor, V. M., Monjas, R., \& Manrique, J. C. (2011). Fifteen years of action research as professional development: seeking more collaborative, useful and democratic systems for teachers. Educational Action Research, 19(2), 153-170.

López-Pastor, V. M., \& Pérez-Pueyo, A. (2017). Buenas prácticas docentes. Evaluación formativa y compartida en educación: Experiencias de éxito en todas las etapas educativas. León: Universidad de León. E-book, en https://buleria.unileon.es/handle/10612/5999

Pascual-Arias, C., López-Pastor, V., \& Hamodi Galán, C. (coord.). (en prensa). Proyecto de innovación docente (PID) y Seminario permanente internivelar sobre Evaluación Formativa y Compartida. Transferencia de conocimiento entre universidad y escuela. XI Congreso Nacional de Evaluación Formativa y Compartida. La evaluación educativa: entre la emoción y la razón.

Pascual Arias, C., Molina Soria, M., Fernández, C., \& Fuentes, T. (2018). Aprendizaje cooperativo, formación permanente del profesorado y evaluación formativa en educación. En Fernández-Rio, J.; Sánchez, R. y Méndez, A. Actas del XI Congreso Internacional de Actividades Físicas Cooperativas (143-154). Oviedo: Universidad de Oviedo. Recuperado de https://congresoafc2018.com/

Santos, M., Martínez, L. F., \& López-Pastor, V. M. (Coords.) (2009). La innovación docente en el EEES. Almería: Editorial Universidad de Almería. 